\title{
SEPARATION AND CONCENTRATION OF ELEMENTS BY REVERSED PHASE PARTITION CHROMATOGRAPHY
}

\author{
I. P. Alimarin and T. A. Bolshova
}

The Chemical Department of M.V. Lomonosov Moscow State University, Moscow, USSR

\begin{abstract}
The method of column extraction chromatography with inert supporting materials facilitates selective or group concentration of traces of elements with an enrichment coefficient more than $10^{5}$ and the separation of complex compounds of elements which have similar properties. Contamination of the solution under investigation is negligible because of slight absorption and the use of small extractant amounts. The method proved to be very convenient for the separation of short-lived isotopes. An additional advantage is the possibility of automatic control of the method.
\end{abstract}

The method of reversed phase partition chromatography is now fairly widely used in radiochemistry, analytical chemistry and nuclear technology for the separation of elements having similar properties. Reversed phase partition chromatography has an undoubted advantage in comparison with ordinary solvent extraction because it allows concentration of traces of elements with high enrichment coefficients. Hence, the method is of particular importance in the analysis of high purity substances used in the radioelectronic industry. An extensive literature deals with the matter ${ }^{1-3}$.

There are two kinds of extraction chromatography depending on which phase, aqueous or organic, is fixed in a column. If a hydrophobic supporting material sorbs a non-polar organic solvent the method is called reversed phase partition chromatography, extraction chromatography or liquidliquid chromatography. A hydrophobic supporting material retains a film of an organic solvent or a solvent-complexing agent. The aqueous solution containing separated elements is the moving phase in this case; the solution passes through the column with the chosen flowrate. We shall not consider here paper chromatography or thin layer chromatography methods.

Hydrophobic silica gel, aluminium oxide, kieselguhr, polytrifluorochloroethylene (Kel-F), and polytetratluoroethylene (teflon) were used as supporting materials. In recent works, some authors have used highly porous teflon ( $\mathrm{PF}-4)$ which has optimum properties as a supporting material for an organic phase ${ }^{4,5}$. The hard structure and porosity of the material facilitate production of a uniformly filled column having a chromatographic system with a greater number of theoretical plates. In general, any organophilic material with hard structure can be used as a support if it does not 
absorb microcomponents or change extractant properties. Teflon is a material of this kind.

Tri- $n$-butylphosphate (TBP) ${ }^{6-9}$, diethylhexylphosphoric acids ${ }^{10-13}$ and other organophosphorus compounds ${ }^{14-17}$, diethylsulphoxide ${ }^{18}$, high molecular weight amines ${ }^{19-21}$, and isobutylmethylketone ${ }^{22}$ have been used fixed on inert supporting materials as extractants to separate and concentrate traces of certain elements. Other work has been devoted to the application of halide and thiocyanate metal complexes with different solvents ${ }^{23-25}$.

Extraction replacement chromatography is a new trend in this field; differing stabilities of the metal chelates give rise to the method. For example, we used exchange reactions of metal diethyldithiocarbamates in chloroform fixed on teflon ${ }^{26}$. This method allowed us to concentrate trace amounts of some metals in the radioactivation analysis of pure yttrium and molybdenum samples ${ }^{27.28}$. Many other exchange reactions of metal chelates dissolved in organic solvents can be used. The extraction chromatography method for metal chelates has been reported ${ }^{29.30}$.

Choice of the best conditions for chromatography makes possible selective isolation of trace elements from complicated mixtures. Column extraction chromatography as a method of separating similar elements has been investigated by a number of chemists. Some separation methods for anionic complexes of metals have been developed ${ }^{31}$. Separation of einsteinium (Es) and californium $(\mathrm{Cf})$ from fermium $(\mathrm{Fm})^{32}$ as well as separations of other transuranium elements have been carried out ${ }^{18}$. The present authors have stressed ${ }^{3}$ the possibility of effective separation of components with close distribution ratios because repeated distribution can take place in a chromatographic column; such separations appeared to be impossible when the ordinary separation funnels were used. This advantage of extraction chromatography permits separation of complexes of close stability. For instance. the authors of rels. 35 and 10 succeeded in concentrating traces of platinum from nickel and manganese after neutron irradiation in sodium permanganate. Chromatographic columns with teflon were used and the enrichment coefficient was $10^{4}$. We achieved ${ }^{2}$ even greater enrichment. about $10^{7}$ to $10^{8}$.

In recent years some work on the elaboration and improvement of the method has been carried out. For exa: iple, the authors of ref. 34 considered the possibility of continuous chromatographic separation of polycomponent mixtures by liquid-liquid chromatography. Many authors have shown that the method cannot be bettered when extremely high purity of the ultimate products and a high degree of enrichment are required.

We shall give here several examples of the application of extraction chromatography for the concentration of traces of elements in the analysis of high purity compounds when macrocomponents are in large excess.

\section{EXPERIMENTAL}

Porous teflon with grain size less than $0.6 \mathrm{~mm}$ was used. The specific surface was $S=6.9 \mathrm{~m}^{2} / \mathrm{g}$. TBP, tri-n-octylphosphate or di-(2-ethylhexyl) phosphoric acid served as the immovable phase.

Teflon was kept in a dryer at $100^{\circ}$ to $110^{\circ} \mathrm{C}$ before use, saturated by an 
organic solvent and placed into a quartz column. The columns used were of $15 \mathrm{~cm}$ length and 3 to $4 \mathrm{~mm}$ in internal diameter. The excess of an extractant was removed by a moving solvent. The column was thermostatically controlled when experimental conditions demanded high temperatures. This latter procedure after filling the column allowed removal of the air from the teflon layer. Increase of temperature in practice did not affect the capacity of one gramme of the immovable phase. The flowrate was between 1 and $0.6 \mathrm{ml} /$ $\min$. The error of the distribution coefficient $(E)$ measurements in different columns but under the same conditions was $\pm 4-5$ per cent.

\section{GROUP AND INDIVIDUAL CONCENTRATION OF ELEMENT TRACES}

We used extraction chromatography for the group concentration of traces of gallium, iron(III), cobalt and copper(II) in the analysis of pure aluminium and its salts and for the separation of indium and cadmium from large amounts of zinc.

Hydrobromic acid was applied as the moving phase for the isolation of indium, cadmium and zinc traces. The values of $E$ for indium, cadmium and zinc increase with increasing concentration of hydrobromic acid in the moving phase. They decrease in the series $\mathrm{Cd}>\mathrm{In}>\mathrm{Zn}$ when the acid concentration is low $(0.3 \mathrm{M})$. Figure 1 shows that this sort of distribution of

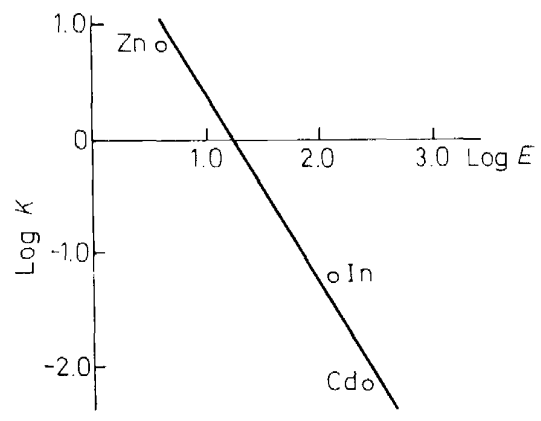

Figure $1 . \log K$ for the bromides of indium, cadmium and zinc as a function of $\log E$.

the elements corresponds to decreases of the non-stability constants of the bromide complexes $\left(K_{1}\right.$ and $\left.K_{2}\right)$. Separation factors $(S)$ are: $S_{\operatorname{In} / \mathbf{Z n}}=31$. $S_{\mathrm{Cd} / \mathrm{Zn}}=56.4, S_{\mathrm{Cd} / \mathrm{In}}=1.8$. The chromatograms of zinc, indium and cadmium separation are given in Figure 2. However, it is necessary to use more concentrated acid ( 0.8 to $1 \mathrm{M}$ ) for the concentration of microamounts of indium. We have been able to separate indium from zinc (ratio $1: 10^{7}$ ) when the concentration of hydrobromic acid was 0.8 to $1 \mathrm{M}$. It is possible to concentrate traces of indium from zinc sulphates and chlorides using $1 \mathrm{M}$ hydrobromic acid as the moving phase. For instance, traces of indium $(2.0$ to $5.0 \mu \mathrm{g})$ were isolated from 10 to $15 \mathrm{~g}$ of zinc sulphate with the error of indium determination \pm 13 per cent. 


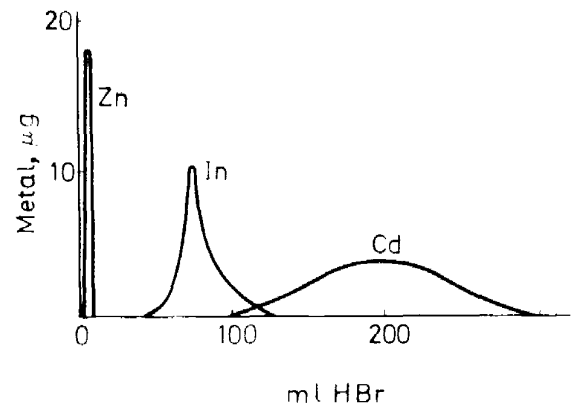

Figure 2. Elution curves of zinc. cadmium and indium from the $0.3 \mathrm{M}$ hydrobromic acid-TBP system. In the column $50 \mu \mathrm{g}$ of each element was added.

The system $2 \mathrm{M}$ hydrobromic acid-tri-(2-ethylhexyl)phosphate was used for the quick chromatographic separation of micro-amounts of indium from comparatively large amounts of gallium. We were able to concentrate $2 \mu \mathrm{g}$ of indium from $2 \mathrm{~g}$ of pure gallium. The enrichment coefficient was $10^{4}$ to $10^{6}$

A molar solution of ammonium thiocyanate as the moving phase and 100 per cent TBP as the immovable one have also been used for the group concentration of some elements. Differences in chromatographic behaviour of certain elements allowed us to concentrate a group of trace elements in the analysis of pure aluminium and its salts (Table $l$ ) as well as in the process of deep purification of ammonium thiocyanate from traces of iron ${ }^{24}$.

\begin{tabular}{|c|c|c|c|}
\hline \multicolumn{4}{|c|}{ Found, " } \\
\hline $\mathrm{Fe}(111)$ & $\mathrm{Ga}(\mathrm{III})$ & $\operatorname{Co}(I I)$ & $\mathrm{Cu}(\mathrm{II})$ \\
\hline $6.5 \times 10 * 6$ & $4.4 \times 10^{-6}$ & $12 \times 10^{-6}$ & $4.4 \times 10^{-6}$ \\
\hline $5.2 \times 10^{-6}$ & $4.6 \times 106$ & $t .3 \times 10^{-6}$ & $4.4 \times 10^{6}$ \\
\hline $5.9 \times 10^{-6}$ & $44 \times 10^{6}$ & $4.5 \times 10^{-6}$ & $4.5 \times 10^{\mathrm{t}}$ \\
\hline $5.1 \times 10^{-6}$ & $4.4 \times 10^{-6}$ & $4.5 \times 10^{-6}$ & $4.5 \times 10$ \\
\hline
\end{tabular}

\section{THE EFFECT OF TEMPERATURE}

It is shown that better extraction separation can be obtained in some cases when the temperature is different from ambient. We found that sometimes a change of temperature allowed us to shorten the time of separation processes and to get narrower peaks. 
In our experiments the temperature was varied between $20^{\circ}$ and $90^{\circ} \pm$ $5^{\circ} \mathrm{C}$. The solutions of $4 \mathrm{M}$ hydrochloric acid, $1 \mathrm{M}$ ammonium thiocyanate or $1 \mathrm{M}$ hydrobromic acid were used as the moving phase.

Figure 3 illustrates the distribution of some elements $(50 \mu \mathrm{g} / \mathrm{ml})$ in the

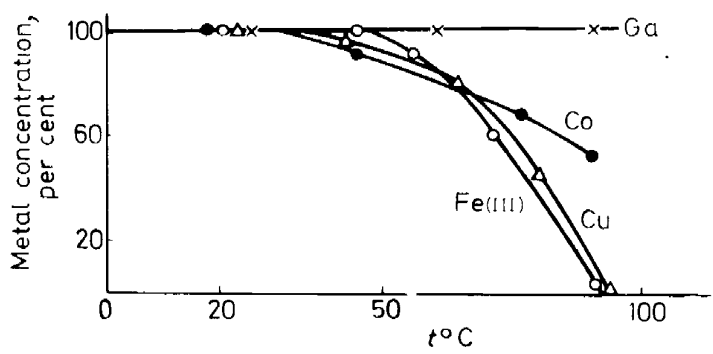

Figure 3. Temperature dependence of metal concentration in the column.

column at different temperatures. It is of interest that an increase of temperature does not affect gallium distribution alone while the content of other elements in the organic phase diminishes with rise of temperature.

For example, iron(III) is sorbed into the organic phase quantitatively if the temperature is $20^{\circ} \mathrm{C}$, by 60 per cent if $t=70^{\circ} \mathrm{C}$ and passes through the column when $t=90^{\circ}$. The process of elution of zinc, cadmium and aluminium is accelerated with increase of temperature. The elution peaks become narrower and consequently the volume of eluate solution necessary for quantitative element elution decreases by several times (Figure 4).

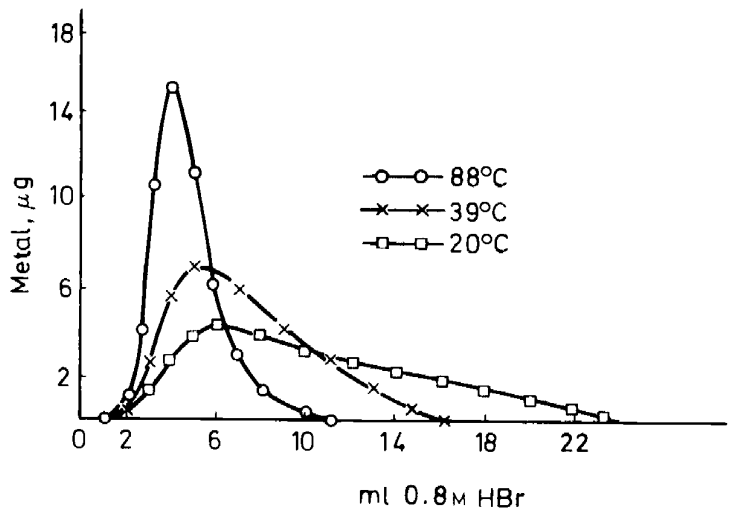

Figure 4. Temperature dependence of elution of zinc from the $0.8 \mathrm{M}$ hydrobromic acid-TBP system.

Investigation of the behaviour of elements at high temperature allowed us to separate small amounts of gallium from comparatively large amounts of iron(III) without addition of ascorbic acid or any other reducing agent. In 


\section{P. ALIMARIN AND T. A. BOLSHOVA}

this case the consumption of the eluate is five times less than at room temperature and the separation procedure takes much less time.

We separated gallium from iron (ratio $1: 10^{5}$ ) in a thermostatically controlled column at $80^{\circ} \mathrm{C}$ in the system $4 \mathrm{~m}$ hydrochloric acid-tributyl phosphate; $20 \mathrm{ml}$ of $4 \mathrm{M}$ hydrochloric acid solution containing $1 \mathrm{~g}$ of $\mathrm{FeCl}_{3} \cdot 6 \mathrm{H}_{2} \mathrm{O}$ and $10 \mu \mathrm{g}$ of gallium were passed through the column tilled with teflon: the flowrate was $0.6 \mathrm{ml} / \mathrm{min}$. Then iron was completely washed by $4 \mathrm{M}$ hydrochloric acid solution (the consumption was about $140 \mathrm{ml}$ ). The process was controlled by the column colour as well as by the reaction with sulphosalicylic acid. Gallium was stripped by $5 \mathrm{ml}$ of $0.1 \mathrm{M}$ hydrochloric acid solution. The error of the determination was less than ten per cent. The separation of gallium from iron can also be carried out from $1 \mathrm{~N}$ solutions of hydrochloric acid at $70^{\circ}-80^{\circ} \mathrm{C}$. Gallium can be separated from large amounts of cadmium in hydrochloric acid solution using thermostatically controlled columns: the separation is impossible at room temperature. Cadmium and indium can also be well separated (Figure 5).

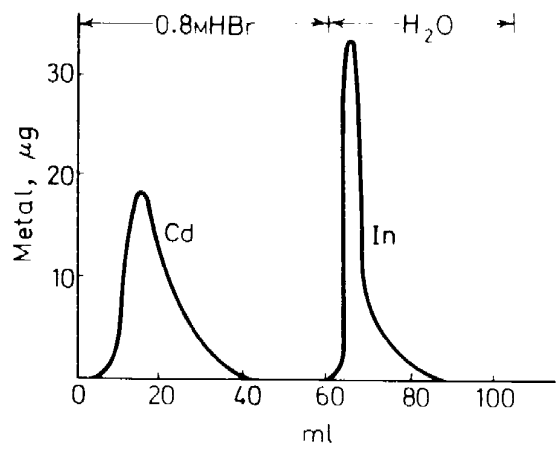

Figure 5. Separation of cadmium from indium $(1: 1)$ at $t=60$

On the other hand, observations show that the extraction of most compounds into the organic phase decreases with increasing temperature. This must be related not with the exothermic nature of the extraction reaction only but with the effect of temperature on the activity coefficient of the complex, complex formation process, dissociation and so on. The effect of temperature on the extraction of complex acids by a hydration solvation mechanism is also a complicated matter.

\section{THE CHANGE OF OXIDATION STATE OF AN ELEMENT}

Recently we suggested a further kind of extraction chromatography based on using specific extraction properties of elements of different oxidation states. 


\section{SEPARATION AND CONCENTRATION OF ELEMENTS}

In the radioactive determination of traces of yttrium, dysprosium, holmium, samarium and lanthanum in europium oxide, europium was determined by its reduction to Eu(II) with metallic zinc in the Jones reductor. The reductor was combined with the teflon chromatographic column saturated by di-(2-ethylhexyl)phosphoric acid (Figure 6). In this case all the europium

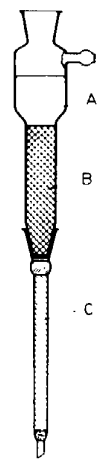

Figure 6. The Jones reductor and chromatograph column-A, solution; B, amalgamated zinc grains; C, powdered PF-4 impregnated with di-(2-ethylhexyl)phosphoric acid.

passed through the column while the traces of other rare earth elements were retained on the column.

The results of the analysis of high purity europium oxide are given in Table 2.

The separation of gallium from large amounts of iron(III) by extraction chromatography can also be carried out after reducing iron to $\mathrm{Fe}(\mathrm{II})$. The concentration factor is $10^{6}$ to $10^{8}$ in this case.

Table 2. Results of $\mathrm{Eu}_{2} \mathrm{O}_{3}$ analysis

\begin{tabular}{lcc}
\hline Element & Content, \% & Sensitivity \\
\hline Yb & $(1.0 \pm 0.2) \times 10^{-5}$ & $1 \times 10^{-6}$ \\
Dy & $(8.0 \pm 0.8) \times 10^{-6}$ & $1 \times 10^{-7}$ \\
Ho & $(4.2 \pm 1.9) \times 10^{-5}$ & $2 \times 10^{-5}$ \\
Sm & $(6.4 \pm 1.1) \times 10^{-5}$ & $2 \times 10^{-7}$ \\
La & $(5.7 \pm 2.4) \times 10^{-5}$ & $1 \times 10^{-5}$ \\
\hline
\end{tabular}

\section{CONCLUSION}

The method of column extraction chromatography with inert supporting materials allows us to carry out selective or group concentrations of traces of elements with an enrichment coefficient of more than $10^{5}$ and to separate 


\section{P. ALIMARIN AND T. A. BOLSHOVA}

complex compounds of elements of similar properties. Contamination of the solutions investigated is negligible because of slight adsorption and the use of small amounts of extractant.

The reagents and water used can be purified beforehand by the same method.

The method proved to be very convenient for the separation of shortlived isotopes. An additional advantage is the possibility of automatic control of the method. This method as well as others can be more efficient if mathematical methods of factor design are used to choose optimum separation conditions.

\section{ACKNOWLEDGEMENT}

The authors thank Dr B. Ya. Spivakov for help with the translation of the text into English.

\section{REFERENCES}

1 G. S. Katykhin, Zh. Analyt. Khim. 20.615(1965).

2 I. P. Alimarin. T. A. Bolshova, N. I. Ershova and M. B. Polinskaja, Zh. Analyt. Khim. 24, 26 (1969).

3 I. P. Alimarin and T. A. Bolshova, Z. Chem. 8, 411 (1968).

4 G. S. Katykhin and M. K. Nikitin, Radiokhimiya, 10, 474 (1968).

5 B. K. Preobrazhenski. A. V. Kalyamin, O. M. Lilova, L. N. Moskwin and B. S. Usikov, Radiokhimiya, 10. 373 and 375 (1968).

'. I. P. Alimarin and T. A. Bolshova, Zh. Analyt. Khim. 21, 411 (1966).

7. P. Alimarin, T. A. Bolshova. N. I. Ershowa and A. D. Kiselewa, Vestnik Moskouskogo Unirersiteta. Ser. II, Khimiya, 4, 55 (1967).

B L, S. Bark, G. Duncan and R. J. T. Graham, Analyst, 92, 347 (1967).

9 L. N. Moskwin, Radiokhimiya, 6, 110 (1964).

10 Yu. G. Zhukowski, G. S. Katykhin, A. L. Martynow and M. K. Nikitin, Radiokhimiya, 10, 252 (1968).

1 E, Herrman, J. Chromatogr. 38, 498 (1968).

12 I. P. Alimarin, T. A. Bolshova, T. G. Dolzhenko and G. M. Kandybina, Zh. Analyt. Khim. 26, $471(1971)$.

13 E. L. Moore and A. Jurriaanse, Analyt. Chem. 39, 733 (1967).

14 I. P. Alimarin, N. I. Ershowa and T. A. Bolshova, Vestnik Moskovskogo Universite'ta. Ser. II, Khimiya, 4, 51 (1967).

15 N. I. Etshowa T. A. Bolschova and I. P. Alimarin, Vestnik Moskovskogo Universitetu. Ser. II, Khimiya, 3, 76 (1968).

is I. P. Alimarin, T. A. Bolshova and N. I. Ershowa, Vestnik Moskonskogo Universiteta, Ser. II, Khimiya, 5, 568 (1970).

17 S. K. Kalinin, G. S. Katykhin, M. N. Nikitin and G. A. Yakowleva, Zh. Analyt. Khim. 23. $1481(1968)$.

18 J. S. Fritz and D. C. Kennedy. Talanta, 17, 837 (1970).

19 R. J. T. Graham, L. S. Bark and D. A. Tisley. J. Chromatogr. 35, 416 (1968).

20) I. M. Gibalo and N. A. Mymrik. Zh. Analyt. Khim. 25, 1747 (1970).

21 I. M. Gibalo and N. A. Mymrik. Zh. Analyt. Khim. 26, 919 (1971).

22 J. S. Fritz., T. Frazee and G. L. Latwesen. Talanta, 17, 857 (1970).

23 S. Przeszlakowski. Chem. Anal. (Warsaw), 12, 1071 (1967).

24 I. P. Alimarin, N. I. Ershowa and T. A. Bolshova. Vestnik Moskonskogo Unitersiteta. Ser. II, Khimiya, 6, 79 (1969).

25 N. I. Ershowa. T. A. Bolshova and I. P. Alimarin. Zh. Analyt, Khim. 26, 243 (1971).

2" O. V. Stepanets, Yu. V. Yakovlev and I. P. Alimarin. Zh. Analyt. Khim. 25, 1906 (1970).

27 I. P. Alimarin and Yu. A. Zolotov. Chem. Anal. (Warsaw), 13, 941 (1968).

28 Yu. A. Zolotov. 'Extraction concentration'. Khimiya: Moscow (1971).

29 T. B. Pierce and P. F. Peck. J. Chromatogr. 6, 248 (1961). 


\section{SEPARATION AND CONCENTRATION OF ELEMENTS}

${ }^{30}$ F. Sebesta. J. Radioanal. Chem. 7, 41 (1971).

31 J. Stronski. Radiochem. Radioanal.Letters, 1, 191 (1969); 5, 113 (1969).

${ }^{32}$ E. P. Horwitz, C. A. A. Bloomquist and D. J. Henderson, J. Inorg. Nucl. Chem. 31, 1149 (1969).

${ }^{33}$ S. K. Kalinin, G. A. Yakovleva, M. K. Nikitin and G. S. Katykhin. 'Gipronikel' Institute Works: Leningrad, 33, 192 (1967).

${ }^{34}$ L. N. Moskvin and L. G. Tsaritsyna. Radiokhimiya, 10, 740 (1968).

35 I. P. Alimarin, A. Z. Miklishanski and Yu. V. Yakovlev. J. Radioanal. Chem. 4, 75 (1970). 